\title{
BIMODULES FOR COMPOSITION ALGEBRAS
}

\section{K. MCCRIMMON}

In this paper we will give a Cayley-Dickson type construction of the bimodules and bimodules with involution for the composition algebras, and then apply these results to the classification of the bimodules for the Jordan matrix algebras.

1. Preliminaries. A composition algebra [1] is a nonassociative algebra $A$ with identity over a field $\Phi$ (always assumed to be of characteristic $\neq 2$ ) which has a nondegenerate quadratic form $Q$ admitting composition

$$
Q(x y)=Q(x) Q(y) .
$$

It is well known that such an algebra is an alternative algebra with involution $x \rightarrow \bar{x}$ such that

$$
x+\bar{x}=T(x) 1, \quad x \bar{x}=\bar{x} x=Q(x) 1 .
$$

There is an explicit procedure for building the composition algebras known as the Cayley-Dickson construction. Given a composition algebra $A$ and a nonzero $\tau \in \Phi$ one defines a multiplication on $A \times A$ by

$$
(x, y) \cdot(z, w)=(x z+\tau \bar{w} y, w x+y \bar{z}) .
$$

If we set $t=(0,1)$ and imbed $A$ by $x \rightarrow(x, 0)$ then we have constructed an algebra

$$
C(A)=A \oplus A t
$$

defined by the relations

$$
\begin{aligned}
x(y t) & =(y x) t, \\
(y t) x & =(y \bar{x}) t, \\
(x t)(y t) & =\tau \bar{y} x .
\end{aligned}
$$

(Strictly speaking, $\boldsymbol{C}(A)$ depends on the choice of $\tau$ as well as on $A$.) We introduce an involution

$$
x+y t \rightarrow \bar{x}-y t \text {. }
$$

Then $Q(u)=u \bar{u}=\bar{u} u$ defines a nondegenerate quadratic form for $u=x+y t \in C(A) ; Q$ admits composition $Q(u v)=Q(u) Q(v)$ if and only if $A$ is associative.

Received by the editors March 17, 1965. 
Starting with the base field $\Phi$ the Cayley-Dickson construction leads to the following tower of composition algebras:

I. $C^{0}(\Phi)=\Phi 1$, the base field.

II. $C^{1}(\Phi)=C\left(C^{0}(\Phi)\right)$, a complex algebra $\Phi 1+\Phi i$ where $i=-i$ and $i^{2}=\lambda 1(\lambda \neq 0)$.

III. $\boldsymbol{C}^{2}(\Phi)=\mathbf{C}\left(\boldsymbol{C}^{1}(\Phi)\right)$, a quaternion algebra with basis $\{1, i, j, k\}$ where $j=-j, i j=-j i=k, j^{2}=\mu 1(\mu \neq 0)$.

IV. $\boldsymbol{C}^{3}(\Phi)=\boldsymbol{C}\left(\boldsymbol{C}^{2}(\Phi)\right)$, a Cayley algebra with basis $\{1, i, j, k, l, i l$, $j l, k l\}$ where $\bar{l}=-l, l^{2}=\nu 1(\nu \neq 0)$.

Since $C^{3}$ is not associative the Cayley-Dickson construction ends here. It can be shown [1] that every composition algebra over $\Phi$ is isomorphic to one of the above algebras (for suitable choice of the parameters $\lambda, \mu, \nu)$. A composition algebra is either a division algebra or else it is split; the split algebras are isomorphic to the algebra obtained by taking all parameters $\lambda, \mu, \nu$ equal to 1 .

A (unital alternative) bimodule for a composition algebra $A$ is a vector space $M$ and two linear maps $L, R: A \rightarrow \operatorname{Hom}(M, M)$ which turn the split null extension

$$
E=A \oplus M
$$

into an alternative algebra with identity $1 \oplus 0$ and multiplication

$$
(a \oplus m)(b \oplus n)=a b \oplus\left(L_{a} n+R_{b} m\right) .
$$

A bimodule with involution is a bimodule $m$ together with an endomorphism $m \rightarrow \bar{m}$ of $M$ of period 2 such that

$$
a \oplus m \rightarrow \bar{a} \oplus \bar{m}
$$

is an involution in $E$.

For example, the regular bimodule and the Cayley-Dickson bimodule are the sub- $A$-bimodules $M=A 1$ and $M=A t$ of $\mathrm{C}(A)$ where $L$ and $R$ are induced by the left and right multiplications of $A$ on $C(A)$ (observe that $A t$ is invariant under $A$ by (2)). There is a second way of representing the Cayley-Dickson bimodule: under the correspondence $a t \leftrightarrow a$ it is equivalent to taking $M=A 1, L_{a}$ and $R_{a}$ the right multiplications in $A$ by $a$ and $\vec{a}$ respectively. Note that the Cayley-Dickson bimodule is alternative if and only if $A$ is associative: $[a t, c, b]+[c, a t, b]$ $=[a, b, c] t$. The regular bimodules with involution $M=(A 1,+)$ and $M=(A 1,-)$ are obtained by taking the regular bimodule $M=A 1$ with the usual involution or its negative. The Cayley-Dickson bimodules with involution $M=(A t,+)$ and $M=(A t,-)$ are obtained 
similarly from the usual involution (namely $-I$ ) induced in the Cayley-Dickson bimodule by the involution in $\boldsymbol{C}(A)$.

2. A lemma. Suppose $M$ is an $A$-bimodule, $m \in M, a \in A$. We claim (3) $a m=m \bar{a} \Rightarrow a(m b)=m(\bar{a} b),(b m) a=(b \bar{a}) m$ for all $b \in A$.

In fact, using (1) $a(m b)-m(\bar{a} b)=a(m b)+m(a b)-T(a) m b$ $=(a m+m a-T(a) m) b=(a m-m \bar{a}) b=0$, and similarly for the other relation. Next we claim

(4) $a m=m a, a^{2}=\lambda 1(\lambda \neq 0) \Rightarrow[a, m, b]=0$, for all $b \in A$.

We have $\lambda[a, m, b]=\lambda(m a) b-a\left(m\left(a^{2} b\right)\right)=\lambda(m a) b-(a m a)(a b)$ $\{$ by the Moufang identity $x(y(x z))=(x y x) z\}=\lambda(m a) b-\left(a^{2} m\right)(a b)$ $=\lambda[m, a, b]=-\lambda[a, m, b]$ by alternativity.

We have the following key

Lemma. Let $M$ be a bimodule for the composition algebra $\mathrm{C}(A)$ $=A \oplus A t, \quad\left\{a_{i}\right\}_{i \in I}, a$ basis for $A$ with $\bar{a}_{i}= \pm a_{i}, Q\left(a_{i}\right) \neq 0, T\left(a_{i} \bar{a}_{j}\right)$ $=0(i \neq j)$.

If $m \in M$ satisfies $a m=m a$ for all $a \in A$ then the element $n=t m$ satisfies $a n=n \vec{a}$ for $a \in A$.

If $m \in M$ satisfies am $=m \bar{a}$ for all $a \in A$ then the elements $n_{i}=\left(a_{i} t\right) m$ $+m\left(a_{i} t\right)$ commute with $\mathbf{C}(A)$,

$$
c n_{i}=n_{i} c \quad \text { for all } c \in C(A),
$$

and the element $n=m+\frac{1}{2} \sum_{I} Q\left(a_{i} t\right)^{-1}\left(a_{i} t\right) n_{i}$ satisfies

$$
c n=n \bar{c} \quad \text { for all } c \in C(A) .
$$

Proof. Our assumptions on the $a_{i}$ imply $a_{i}^{2}=\lambda_{i} 1$ for $\lambda_{i}= \pm Q\left(a_{i}\right)$ $\neq 0$. For the first part we may take $a=a_{i}$ and apply associativity (4) to obtain $a n=a(t m)=a t m=t \bar{a} m=(t m) \bar{a}=n \bar{a}$; since this holds for a basis it holds for all $a \in A$.

For the second part it suffices to prove the relations for $c=a_{j}$ and $c=a_{j} t$. First consider $c=a_{j}$ and set $d=a_{i} t$. Then $c n_{i}=c(d m+m d)$ $=c(d m)+m(\bar{c} d)($ by $(3))=c(d m)+m(d c)$. Dually $n_{i} c=(c d) m+(m d) c$, so by alternativity

$$
c n_{i}=n_{i} c .
$$

From this and (4) we conclude $c\left(d n_{i}\right)=c d n_{i}=d \bar{c} n_{i}=\left(d n_{i}\right) \bar{c}$, and putting this together with $c m=m \bar{c}$ gives

$$
c n=n \bar{c} .
$$

Now consider $c=a_{j} t$, with $d=a_{i} t$ as before. Then $c \in A d$, and $\left[a d, n_{i}\right]$ 
$=a\left[d, n_{i}\right]$ by (4) and the above, so from $\left[d, n_{i}\right]=[d, d m+m d]$ $=\left[d^{2}, m\right]=-Q(d)[1, m]=0$ we conclude

$$
c n_{i}=n_{i} c .
$$

Hence by (4) $c\left(d n_{i}\right)-\left(d n_{i}\right) \bar{c}=(c d-d \bar{c}) n_{i}=(c d+d c) n_{i}=-2 \delta_{i j} Q\left(a_{j} t\right) n_{j}$. Combining this with $c m-m \bar{c}=c m+m c=n_{j}$ gives

$$
c n-n \bar{c}=0 \text {. }
$$

3. Bimodules. We now give a Cayley-Dickson type construction of the bimodules for the composition algebras. We remark that the sub-bimodules of the regular $A$-bimodules are the two-sided ideals of $A$, so the regular bimodules are irreducible except for the case of the split $C^{1}$, which splits into a direct sum $C^{1}=\Phi e_{1} \oplus \Phi e_{2}$ of fields where $e_{1}=\frac{1}{2}(1+i), e_{2}=\bar{e}_{1}=\frac{1}{2}(1-i)\left(i^{2}=1\right)$. The second way of representing the Cayley-Dickson bimodule shows that the sub-bimodules correspond to the right ideals of $A$, so the Cayley-Dickson bimodules are irreducible except for the case of the split $\boldsymbol{C}^{1}$ or $\boldsymbol{C}^{2}$, where it splits into a direct sum $A t=\left(e_{1} A\right) t \oplus\left(e_{2} A\right) t$ of two irreducible bimodules. (Even though the split $C^{3}$ is not a division algebra, it has no proper right ideals.) In all cases the regular and Cayley-Dickson bimodules are completely reducible.

Theorem 1. Every bimodule $M$ for a composition algebra $A$ is completely reducible. The irreducible bimodules are isomorphic to the irreducible sub-bimodules of the regular and Cayley-Dickson bimodules. Thus we have the following list of irreducible bimodules.

I. $A=C^{0}: M=A 1$.

IIa. $A=C^{1}$ division algebra: $M=A 1, M=A j$.

IIb. $A=C^{1}$ split: $M=\Phi e_{1}, M=\Phi e_{2}, M=\Phi e_{1} j, M=\Phi e_{2} j$.

IIIa. $A=C^{2}$ division algebra: $M=A 1, M=A l$.

IIIb. $A=C^{2}$ split: $M=A 1, M=\Phi e_{1} l+\Phi\left(e_{1} j\right) l$.

IV. $A=C^{3}: M=A 1$.

Proof. The above list results from the fact that $A 1 \cong A i$ for $A=C^{0}$, that $\left(e_{1} A\right) l \cong\left(e_{2} A\right) l$ under $a l \leftrightarrow(j a) l$ for $A=C^{2}$, and that $A t$ is not alternative for $A=C^{3}$. It is enough to show that every cyclic bimodule $M=\{m\}$ is a sum of homomorphic images of regular and Cayley-Dickson bimodules. If $a m=m a$ for all $a \in A$ then $\phi: a \rightarrow a m$ is a homomorphism of the regular bimodule $A 1$ onto $\{m\}$ since for basis elements $a=a_{i}$ as in the lemma we have $\phi\left(R_{a} b\right)=(b a) m=(b m) a$ $=R_{a} \phi(b)$ (using (4)), $\phi\left(L_{a} b\right)=a(b m)=L_{a} \phi(b)$. Similarly if $a m=m \bar{a}$ for all $a \in A$ then $\psi: a t \rightarrow a m$ is a homomorphism of the Cayley-Dickson bimodule $A t$ onto $\{m\}$ since by $(3) \psi\left(R_{a}(b t)\right)=\psi((b \bar{a}) t)=(b \bar{a}) m$ 
$=(b m) a=R_{a} \psi(b), \psi\left(L_{a}(b t)\right)=\psi((b a) t)=(b a) m=m(\bar{a} \bar{b})=a(m \bar{b})=a(b m)$ $=L_{a} \psi(b)$. Hence the problem is reduced to proving that $\{m\}$ can be generated by elements of the above two kinds.

I. We have $A \supset \mathbf{C}^{0}=\Phi 1.1^{0}=m$ has $\{m\}=\left\{1^{0}\right\}$ and satisfies $c 1^{0}=1^{\circ} c$ for all $c \in C^{0}$, so if $A=C^{0}$ we are done.

II. If $A \neq C^{0}$ then $A \supset \boldsymbol{C}^{1}=\boldsymbol{C}^{0}+C^{0} i$. Given $1^{0}$ as above we set $1^{1}=i 1^{0}+1^{0} i, j^{1}=1^{0}+\frac{1}{2} Q(i)^{-1} i 1^{1}$, so $\left\{1^{0}\right\}=\left\{1^{1}, j^{1}\right\}$ and by the lemma, $c 1^{1}=1^{1} c, c j^{1}=j^{1} \bar{c}$ for all $c \in C^{1}$. If $A=C^{1}$ we are done.

III. If $A \neq C^{1}$ then $A \supset C^{2}=C^{1}+C^{1} j$. Given $j^{1}$ as above we have $\left\{j^{1}\right\}=\left\{1_{j}^{2}, 1_{k}^{2}, l^{2}\right\}$ for $1_{j}^{2}=j j^{1}+j^{1} j, 1_{k}^{2}=k j^{1}+j^{1} k, l^{2}=j^{1}+\frac{1}{2} Q(j)^{-1} j 1_{j}^{2}$ $+\frac{1}{2} Q(k)^{-1} k 1_{k}^{2}$. By the lemma, $c 1_{j}^{2}=1_{j}^{2} c, c 1_{k}^{2}=1_{k}^{2} c, c l^{2}=l^{2} \bar{c}$ for all $c \in C^{2}$. Similarly, given $1^{1}$ as above we can define $j^{1}=j 1^{1}$; by the first part of the lemma $a j^{1}=j^{1} \bar{a}$ for $a \in C^{1}$, so we get $\left\{1^{1}\right\}=\left\{j^{1}\right\}=\left\{1_{j}^{2}, 1_{k}^{2}, l^{2}\right\}$ as before. If $A=C^{2}$ we are done.

IV. If $A \neq C^{2}$ then $A=C^{3}=C^{2}+C^{2} l$. Given $l^{2}$ as above we have $\left\{l^{2}\right\}=\left\{1_{l}^{3}, 1_{i l}^{3}, 1_{j l}^{3}, 1_{k l}^{3}, t^{3}\right\}$ for $1_{a l}^{3}=(a l) l^{2}+l^{2}(a l)(a=1, i, j, k), t^{3}=l^{2}$ $+\frac{1}{2} Q(l)^{-1} l 1_{l}^{3}+\frac{1}{2} Q(i l)^{-1}(i l) 1_{i l}^{3}+\frac{1}{2} Q(j l)^{-1}(j l) 1_{j l}^{3}+\frac{1}{2} Q(k l)^{-1}(k l) 1_{k l}^{3}$. By the lemma, $c 1_{a l}^{3}=1_{a l}^{3} c, c t^{3}=t^{3} \bar{c}$ for $c \in C^{3}=A$. Similarly, given $1^{2}$ as above we set $l^{2}=l 1^{2}$; by the first part of the lemma $a l^{2}=l^{2} \bar{a}$ for $a \in C^{2}$ so we can repeat as before to obtain $\left\{1^{2}\right\}=\left\{l^{2}\right\}=\left\{1_{l}^{3}, 1_{i l}^{3}, 1_{j l}^{3}, 1_{k l}^{3}, t^{3}\right\}$. Since $A=C^{3}$ we are done.

4. Bimodules with involution. Every element of a bimodule with involution is the sum of its symmetric and skew parts. If we start the procedure of Theorem 1 with a symmetric or sliew $m$, and note that the involution on $\{m\}$ is completely determined by $\bar{m}$, we arrive at the following

THEOREM 2. Every bimodule $M$ with involution for the composition algebra $A$ is completely reducible. The irreducible bimodules with involution are isomorphic to the irreducible sub-bimodules of the regular and Cayley-Dickson bimodules with involution. We thus obtain the following list.

I. $A=C^{0}: M=(A 1,+), M=(A 1,-)$.

IIa. $A=C^{1}$ division algebra: $M=(A 1,+), M=(A j,+)$, $M=(A j,-)$.

IIb. $A=C^{1}$ split: $M=(A 1,+), M=\left(\Phi e_{1} j,+\right), M=\left(\Phi e_{1} j,-\right)$, $M=\left(\Phi e_{2} j,+\right), M=\left(\Phi e_{2} j,-\right)$.

IIIa. $A=C^{2}$ division algebra: $M=(A 1,+), M=(A 1,-), M$ $=(A l,+), M=(A l,-)$.

IIIb. $A=C^{2}$ split: $M=(A 1,+), M=(A 1,-)$, $M=\left(\Phi e_{1} l+\Phi\left(e_{1} j\right) l,+\right), M=\left(\Phi e_{1} l+\Phi\left(e_{1} j\right) l,-\right)$.

IV. $A=C^{3}: M=(A 1,+), M=(A 1,-)$. 
The difference from the list in Theorem 1 is due to the fact that for $A=C^{1}$ we have $(A 1,+) \cong(A 1,-)$ under $a \rightarrow i a$; this is irreducible as bimodule with involution even in the split case.

5. Bimodules for Jordan algebras. From N. Jacobson's Coordinatization Theorem [2] it follows that for $n \geqq 3$ the unital bimodules for a simple Jordan matrix algebra $H\left(A_{n}, \gamma\right.$ ) (where $A$ is a composition algebra and $H\left(A_{n}, \gamma\right)$ is the subalgebra of $A_{n}^{+}$consisting of the symmetric matrices relative to the canonical involution $\left.X \rightarrow \gamma^{-1} \bar{X}^{\prime} \gamma\right)$ are the bimodules $H\left(M_{n}, \gamma\right)$ where $M$ is a unital bimodule for $A$ which is associative if $n \geqq 4$ and in any case is alternative with involution whose selfadjoint elements are in the nucleus.

For example, the regular Jordan bimodules for $H\left(A_{n}, \gamma\right)$ are $H\left((A 1,+)_{n}, \gamma\right)$, which is just $H\left(A_{n}, \gamma\right)$ itself, and $H\left((A 1,-)_{n}, \gamma\right)$, which is the set $S\left(A_{n}, \gamma\right)$ of matrices skew under $X \rightarrow \gamma^{-1} \bar{X}^{\prime} \gamma$. The method of representing the Cayley-Dickson bimodules $M=A t$ as $M=A$ with $L_{a}, R_{a}$ the right multiplications by $a, \bar{a}$ allows us to interpret $H\left((A t,+)_{n}, \gamma\right)$ and $H\left((A t,-)_{n}, \gamma\right)$ as the Cayley-Dickson Jordan bimodules $\mathrm{CS}\left(A_{n}, \gamma\right)=\left\{X \in A_{n} \mid \gamma^{-1} X^{\prime} \gamma=-X\right\}$ and $\mathrm{CH}\left(A_{n}, \gamma\right)$ $=\left\{X \in A_{n} \mid \gamma^{-1} X^{\prime} \gamma=X\right\}$ where the module operation is $H \cdot X$ $=\frac{1}{2}\left\{\left(X^{\prime} H^{\prime}\right)^{\prime}+X \bar{H}\right\}$ for $H \in H\left(A_{n}, \gamma\right)$. (Note that if $A$ is commutative this is just $H \cdot X=\frac{1}{2}(H X+X \bar{H})$.) If $A$ is a split $C^{1}$ or $C^{2}$ these are not irreducible; we have $C S\left(A_{n}, \gamma\right)=C S\left(\left(e_{1} A\right)_{n}, \gamma\right) \oplus C S\left(\left(e_{2} A\right)_{n}, \gamma\right)$ $=C_{1} S\left(A_{n}, \gamma\right) \oplus C_{2} S\left(A_{n}, \gamma\right)$, and similarly

$$
\mathrm{C} H\left(A_{n}, \gamma\right)=\mathrm{C}_{1} H\left(A_{n}, \gamma\right) \oplus \mathrm{C}_{2} H\left(A_{n}, \gamma\right),
$$

where the $\boldsymbol{C}_{\boldsymbol{i}}$ are irreducible. (In the commutative case of a split $\boldsymbol{C}^{1}$ we may identify $e_{i} A=\Phi e_{i}$ with $\Phi$; writing $H=H_{1} e_{1}+H_{2} e_{2}$ for $H_{i} \in \Phi_{n}$, and noting $\bar{H}=H_{2} e_{1}+H_{1} e_{2}$, the modules $C_{1}$ and $C_{2}$ correspond to the tow actions $H \cdot X=\frac{1}{2}\left(H_{1} X+X H_{2}\right)$ and $H \cdot X=\frac{1}{2}\left(H_{2} X+X H_{1}\right)$ of $H$ on $\left.X \in \Phi_{n}.\right)$

Theorem 3. Every unital bimodule $N$ for the simple Jordan algebra $H\left(A_{n}, \gamma\right)(n \geqq 3, A$ a composition algebra) is completely reducible. The irreducible bimodules are isomorphic to the irreducible sub-bimodules of the regular and Cayley-Dickson Jordan bimodules. Thus we arrive at the following list.

I. $A=\mathbf{C}^{0}: N=H\left(A_{n}, \gamma\right), N=S\left(A_{n}, \gamma\right)$.

IIa. $A=C^{1}$ division algebra: $N=H\left(A_{n}, \gamma\right), \quad N=\mathrm{CH}\left(A_{n}, \gamma\right)$, $N=\operatorname{CS}\left(A_{n}, \gamma\right)$.

IIb. $A=C^{1}$ split: $N=H\left(A_{n}, \gamma\right), N=C_{1} H\left(A_{n}, \gamma\right), N=C_{1} S\left(A_{n}, \gamma\right)$, $N=C_{2} H\left(A_{n}, \gamma\right), N=C_{2} S\left(A_{n}, \gamma\right)$. 
IIIa. $A=C^{2}$ division algebra: $N=H\left(A_{n}, \gamma\right), N=S\left(A_{n}, \gamma\right)$, and if $n=3$ also $N=C S\left(A_{3}, \gamma\right)$.

IIIb. $A=C^{2}$ split: $N=H\left(A_{n}, \gamma\right), N=S\left(A_{n}, \gamma\right)$, and if $n=3$ also $N=C_{1} S\left(A_{3}, \gamma\right)$.

IV. $A=C^{3}(n=3): N=H\left(A_{3}, \gamma\right)$.

The simplification in the list of Theorem 2 comes from the fact that in the bimodules $S\left(A_{n}, \gamma\right)=H\left((A 1,-)_{n}, \gamma\right)$ for $C^{3}$ and $C H\left(A_{n}, \gamma\right)$ $=H\left((A l,-)_{n}, \gamma\right)$ for $\boldsymbol{C}^{2}$ the underlying alternative bimodules $(A 1,-)$ and $(A l,-)$ do not have all selfadjoint elements in the nucleus, and in $C S\left(A_{n}, \gamma\right)=H\left((A l,+)_{n}, \gamma\right)$ for $C^{2}$ the underlying alternative bimodule $(A l,+)$ is not associative.

The results of Theorems 1 and 3 are due to N. Jacobson [3] by a somewhat longer method using the universal enveloping algebras of the birepresentations.

\section{BIBLIOGRAPHY}

1. N. Jacobson, Composition algebras and their automorphisms, Rend. Circ. Mat. Palermo 7 (1958), 55-80.

2. - A coordinatization theorem for Jordan algebras, Proc. Nat. Acad. Sci. U.S.A. 48 (1962), 1154-1160. $1-71$.

3. - Structure of alternative and Jordan bimodules, Osaka Math. J. 6 (1954),

YALE University 